\title{
Effect of Structural Disorder on the Electronic Density of States in One-Dimensional Chain of Atoms
}

\author{
Maciej Wołoszyn and Bartłomiej J. Spisak \\ Faculty of Physics and Nuclear Techniques, \\ AGH-University of Science and Technology, \\ Al. Mickiewicza 30, \\ 30-059 Kraków, Poland \\ woloszyn@novell.ftj.agh.edu.pl
}

\begin{abstract}
A simple model of chain containing Dirac's delta-like potentials is used to calculate the density of states in systems with structural disorder. The crystal case of equally spaced scattering centers may be seen as a reference case suitable both for numerical calculations as well as analytical ones. The structural disorder means distortion of ideal lattice seen as fluctuation of distances between adjacent sites. Such disorder is sometimes referred to as amorphous and may lead to smearing off the band boundaries resulting in a tail-like density of states.
\end{abstract}

\section{Introduction}

In the last decade we observe a great interest in the low dimensional systems. The rapid development of nanotechnology allows to fabricate the new class of artificial structures such as dots, wires and wells, where the motion of electrons is confined by the spatial dimensions of the system [1. Therefore the dots, the wires and the wells are regarded as zero-, one- and two- dimensional systems, respectively.

The electronic structure of these systems is especially important for practical applications and its theoretical analysis can be based on the Bethe ansatz method 2], the concept of low energy Tomonaga-Luttinger liquid [3] and powerful numerical techniques such as the Quantum Monte-Carlo [4] or Density Matrix Renormalization Group Method [5]. Another important numerical techniques are Coherent Potential Approximation [6] and Recursion Method [7]. Especially the last two methods allow us to construct the density of states (DoS) [8] in a simple manner.

As it is well known the structural disorder, i.e. spatial fluctuations of potential, has a strong influence on the electronic phenomena in condensed matter [9]. In this paper we adopt the generalized Kronig-Penney model to the description of the chain of atoms (which can be treated as a model of quantum wire) and confine our attention to the modification of the DoS as a result of amorphisation. 


\section{Model}

In the presented simple model which can be regarded as a generalization of the Kronig-Penney model [10, we consider the time-independent Schrödinger equation for the one-dimensional system of noninteracting electrons with effective mass $m$ which move in a random potential $V(x)$, namely

$$
\left[-\frac{\hbar^{2}}{2 m} \frac{\mathrm{d}^{2}}{\mathrm{~d} x^{2}}+V(x)\right] \psi(x)=\varepsilon \psi(x),
$$

where $\psi(x)$ is the wave function, $\varepsilon$ - energy and $\hbar$ - the Planck constant. In fact, the potential $V(x)$ represents the collection of $N$ atoms and consequently scattering potentials well located at random positions $x_{i}$, namely

$$
V(x)=\sum_{i=1}^{N} F_{0} \frac{\hbar^{2}}{m} \delta\left(x-x_{i}\right),
$$

where $F_{0}=\hbar^{2} V_{0} b / m$ and parameter $b$ is defined as the width of the square interatomic barriers with $V_{0}$ being their height. The distribution of these scattering centers in the chain can be described by the probability distribution function, $P\left\{x_{i}\right\}$, for the random potential [11].

The limit $V_{0} \rightarrow \infty$ and $b \rightarrow 0$ allows us to define the constant strength of scattering potential, i.e. $V_{0} b=$ const, and even in the case of disordered chain of atoms we can construct the wave function of electrons which satisfies (1), using the analytical expression for the wave function $\psi_{i}(x)$ inside the $i$-th square well

$$
\psi_{i}(x)=A_{i} \cos \left(u x+\xi_{i}\right),
$$

where $A_{i}$ is the amplitude, $u=\sqrt{2 m \varepsilon} / \hbar$ and $\xi_{i}$ is the phase.

Joining solutions (3) found at consecutive ranges gives the wave function $\psi(x)$ which must satisfy the following conditions:

$$
\begin{gathered}
\psi\left(x_{i}^{+}\right)=\psi\left(x_{i}^{-}\right), \\
\left.\frac{\mathrm{d}}{\mathrm{d} x} \psi(x)\right|_{x_{i}^{+}}-\left.\frac{\mathrm{d}}{\mathrm{d} x} \psi(x)\right|_{x_{i}^{-}}=2 F_{0} \psi\left(x_{i}\right),
\end{gathered}
$$

where the finite discontinuity at the step results from integrating (1).

Of course the boundary values $\psi\left(x_{1}\right)$ and $\psi\left(x_{N}\right)$ also have to be provided to calculate $A_{i}$ and $\xi_{i}$ for each $i$-th $(i=1, \ldots, N-1)$ potential well, which is achieved by applying conditions (4) and (5) at each site $x_{i}$.

The knowledge of exact form of $\psi(x)$ allows us to find the DoS function $\rho(E)$ from the relation

$$
\rho(E)=\frac{\mathrm{d} S}{\mathrm{~d} E},
$$

where $S(E)$ is the number of zeros of the wave function $\psi(x)$ and corresponds to the number of excited states with energy $E \leq \varepsilon$. Since each function $\psi_{i}(x)$ has 
the simple form of a cosine finding all zeros is quite straightforward, however the whole procedure has to be repeated for all values of energy $E$ for which we intend to obtain the DoS function. It allows us to calculate $S(E)$ and than the DoS from (6).

\section{Results and Conclusions}

The method described above provides the way to find the DoS function for disordered systems with $x_{i+1}=x_{i}+\bar{a}+\eta \Delta x$. The average distance between neighboring sites is denoted as $\bar{a}=\sum_{i=1}^{N} a_{i}$ where $a_{i}=x_{i+1}-x_{i}$.

$\eta$ is a random value from the range $(-1 ; 1)$ and $\Delta x$ describes the degree of disorder. For calculations presented in this paper we assume the average distance between neighboring sites $\bar{a}=1$, number of sites $N=10^{4}$ and $F_{0}=1$ with arbitrary units.

For $\Delta x=0$ we obtain a fully ordered chain of potentials corresponding to a pure crystal and the $\rho(E)$ function for that system is presented on all figures for reference with dashed line. It shows well known characteristics of the DoS function for one-dimensional ordered systems, that is $\rho(E) \propto E^{-1 / 2}$ at the edge of each band.

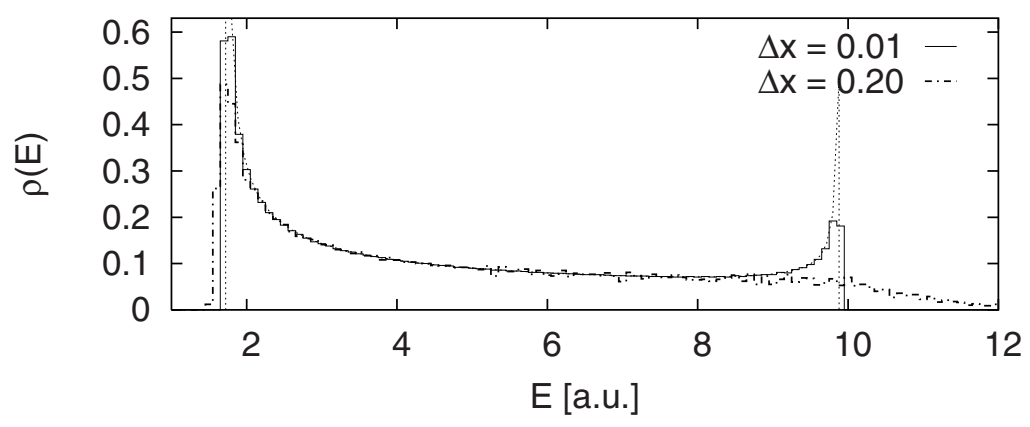

Fig. 1. DoS $\rho(E)$ for different values of $\Delta x: 0.01$ and 0.20 . For reference, thin dashed line shows the DoS of the ordered system with the same $F_{0}$ and all $a_{i}=\bar{a}$

In case of disordered systems the characteristic deformation of the DoS function is observed (Fig. 1): previously sharp edges of the electronic bands smear off forming so-called "tails", where the localized states are expected (the problem of localization will be discussed in the forthcoming paper).

Broadening of the energy bands leads as it should be expected to filling the gaps when the bands start to overlap. The results on Fig. 2 show the vanishing gap between the first and the second band for the growing disorder.

It is well known that for example amorphous silicon exhibits band structure with the DoS showing characteristic "tails" which extend beyond the originally 


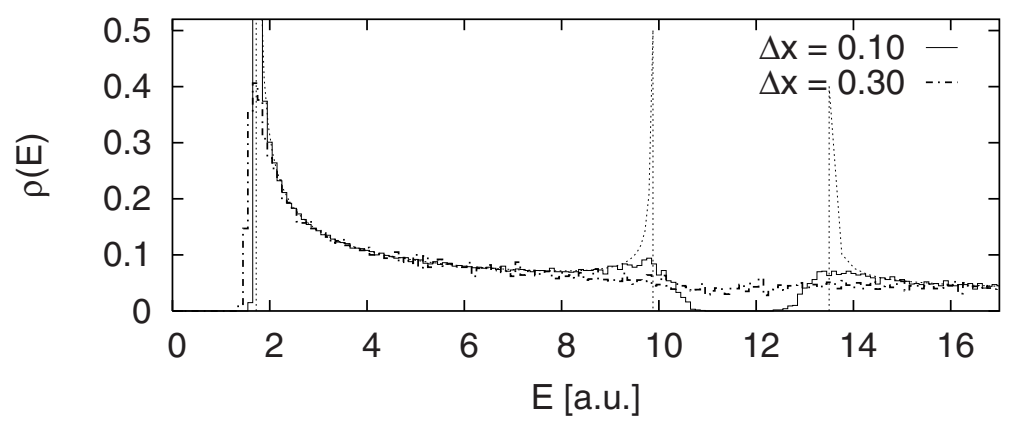

Fig. 2. The gap (or lack of it) in the DoS function $\rho(E)$ for various degrees of disorder: $\Delta x=0.10$ and $\Delta x=0.30$. Thin dashed line denotes the DoS of the ordered system with the same $F_{0}$ and all $a_{i}=\bar{a}$

placed band boundary for the crystal 12. These tails correspond to localized states and so amorphisation may serve as an alternative possibility to introduce localized scattering centers, as it is known for the amorphous semiconducting silicon, instead of the standard doping technique.

The discussed computer simulation may help for the better understanding of how the amorphisation described in terms of parameter $\Delta x$ alters the DoS. In the computer experiment we scan $\Delta x$ from zero, the reference case of a pure crystal when the computer simulation restores the known analytical results, to larger $\Delta x>0$ when we may observe how Dos reacts to the amorphisation. In particular, we report (a) the sharp boundary is smeared off when $\Delta x$ increases and (b) the gap between bands even vanishes for $\Delta x$ above a critical value.

\section{References}

1. Ando, T., Fowler, A.B., Stern, F. Rev. Mod. Phys. 54 (1982) 437

2. Castro-Alvaredo, O., Fring, A. Nucl. Phys. B 649 (2003) 449

3. Mahan, G.D.: Many-Particle Physics. Kluwer Academic/Plenum Publishers, New York, Boston,Dordrecht, London, Moscow (2000)

4. Evertz, H.G. Adv. Phys. 52 (2003) 1

5. Peschel, I., Wang, X., Kaulke, M., Hallberg, K., eds.: Density-Matrix Renormalization, A New Numerical Method in Physics. Springer, Berlin (1999)

6. Wołoszyn, M., Maksymowicz, A. TASK Quarterly 6 (2002) 669

7. Haydock, R. In Ehrenreich, H., Seitz, F., Turnbull, D., eds.: Solid State Phys. Volume 35. Academic Press, London (1980) 216

8. Ziman, J.M.: Principles of the Theory of Solids. Cambridge University Press, London (1972)

9. Anderson, P.W. Phys. Rev. 109 (1958) 1492

10. Kronig, R. de L., Penney, W.G. Proc. Roy. Soc. A130 (1930) 499

11. Economou, E.N.: Green's Functions in Quantum Physics. Springer-Verlag, BerlinHeidelberg-New York-Tokyo (1983)

12. Aljishi, S., Cohen, J.D., Jin, S., Ley, L. Phys. Rev. Lett. 64 (1990) 2811 